\title{
Liver Abscess in a Pediatric Patient with Ulcerative Colitis: A Case Presentation
}

\author{
Daniel F. Castillo*1, Ricardo Caicedo ${ }^{2}$ and Vani Gopalareddy ${ }^{2}$ \\ ${ }^{1}$ Department of Pediatrics, Atrium Health- Levine Children's Hospital, Charlotte, NC, USA; ${ }^{2}$ Department of Pediatric \\ Gastroenterology, Atrium Health- Levine Children's Hospital, Charlotte, NC, USA
}

\begin{abstract}
Hepatic abscesses are an uncommon extra-intestinal manifestation of inflammatory bowel disease, the incidence of which has been estimated to be approximately 7 per 10,000 patients with inflammatory bowel disease. It is unclear whether patients with Crohn's disease or patients with ulcerative colitis (UC) are at higher risk of developing this complication. Based on case reports, most cases are found in Crohn's disease; however, a recent cohort study showed an increased risk in UC instead. Hepatic abscesses in the pediatric population are rare, and there have been no reported cases of hepatic abscesses in a pediatric patient with UC. We describe herein a pediatric patient with UC who developed hepatic abscesses and portal vein thrombosis. This patient also had an extended time in remission from his UC despite being off of immunosuppressive therapies, and we speculate on how his clinical course and treatment strategies may have contributed to this.

Citation of this article: Castillo DF, Caicedo R, Gopalareddy V. Liver abscess in a pediatric patient with ulcerative colitis: A case presentation. J Clin Transl Hepatol 2019;7(1):93-96. doi: 10.14218/JCTH.2018.00045.
\end{abstract}

\section{Case report}

A 16 year-old male presented with fever, fatigue, scleral icterus, jaundice, dark urine, and right abdominal and right shoulder pain. He had been diagnosed with ulcerative colitis (UC) 2 years prior but otherwise did not have any significant medical history. From diagnosis, he had taken a corticosteroid-dependent course and experienced several acute exacerbations of his disease, prompting escalation of therapy from aminosalicylates to immunomodulators. He completed a three-dose induction course of infliximab 1 month prior to admission. His latest UC flare was approximately 3 weeks before presentation, with a colonoscopy showing pancolitis. He was treated with IV corticosteroids and another dose of infliximab, after which he improved. His alkaline phosphatase (ALP) at that time was $88 \mathrm{U} / \mathrm{L}$, within normal limits (Fig. 1).

Keywords: Ulcerative colitis; IBD; Liver abscess.

Abbreviations: ALP, alkaline phosphatase; ALT, alanine aminotransferase; AST, aspartate aminotransferase; CRP, C-reactive protein; CT, computed tomography; IBD, inflammatory bowel disease; UC, ulcerative colitis.

Received: 28 July 2018; Revised: 27 December 2018; Accepted: 7 January 2019 *Correspondence to: Daniel F. Castillo, Department of Pediatrics, Atrium HealthLevine Children's Hospital, 1000 Blythe Blvd, Charlotte, NC 28203, USA. Tel: +1407-314-5294, Fax: +1-704-381-6841, E-mail: daniel.castillo@atriumhealth.org
On presentation, the patient had leukocytosis $(16,000 /$ $\mu \mathrm{L})$, anemia $(6.9 \mathrm{~g} / \mathrm{dL})$, thrombocytopenia $(53,000 / \mu \mathrm{L})$, transaminitis (aspartate aminotransferase [AST] $570 \mathrm{U} / \mathrm{L}$, alanine aminotransferase [ALT] $296 \mathrm{U} / \mathrm{L})$, a highly elevated $\operatorname{ALP}(426 \mathrm{U} / \mathrm{L})$, and hyperbilirubinemia $(3.1 \mathrm{mg} / \mathrm{dL})$. His C reactive protein (CRP) was $20.4 \mathrm{mg} / \mathrm{dL}$. Computed tomography (CT) showed multiple multi-loculated abscesses in the right hepatic lobe, the largest in segment 7 that measured approximately $10 \mathrm{~cm}$ and the presence of gas (Fig. 2). The CT also showed extensive intrahepatic thrombosis of the right portal vein, splenomegaly, bilateral pleural effusions, and diffuse colonic wall thickening. However, there was no megacolon, no free intraperitoneal air and no other signs of perforation on the $\mathrm{CT}$, and the appendix was commented on as being normal.

The abscess was initially drained by interventional radiology, and cultures were positive for Peptostreptococci, skin flora (not specified, described as Gram-positive cocci in clusters and Gram-positive cocci in pairs, suggesting staphylococcal and possibly streptococcal species), and Escherichia coli. An acid-fast bacilli stain was done on this aspirate, and gave a negative result. The patient was also bacteremic initially with Streptococcus viridans and then E. coli. Because of the size of the abscesses, surgical drainage was required; repeat wound cultures showed the same organisms.

The patient developed septic shock post-operatively and required ICU management. Throughout this stay, based on sensitivities, antimicrobial therapy was changed from vancomycin and meropenem to ceftriaxone and metronidazole. Repeat CT (10 and then 20 days after the initial study) showed continued improvement in size of the abscesses over the next month. Therapy was again de-escalated to oral amoxicillin-clavulanate, of which he completed a 2-week course.

As the patient had symptomatically improved and his CRP was down-trending, he was discharged on an amino acidbased formula via nasogastric tube and advancing oral diet. The liver loculations improved and resolved on ultrasonography by 6 months from discharge, with normalization of liver enzymes and inflammatory markers at that time as well. Throughout this time, he was on no UC therapy other than exclusive enteral nutrition, with which he was not consistently compliant. He remained without signs or symptoms of UC until approximately 11 months from admission when he developed severe pancolitis for which he underwent a total colectomy.

Surgical gross pathology showed moderately to severely active chronic pancolitis with mural abscesses; the mucosa was diffusely tan-red and hyperemic, with scattered diffuse 
Castillo D.F. et al: Liver abscess in a pediatric patient with UC

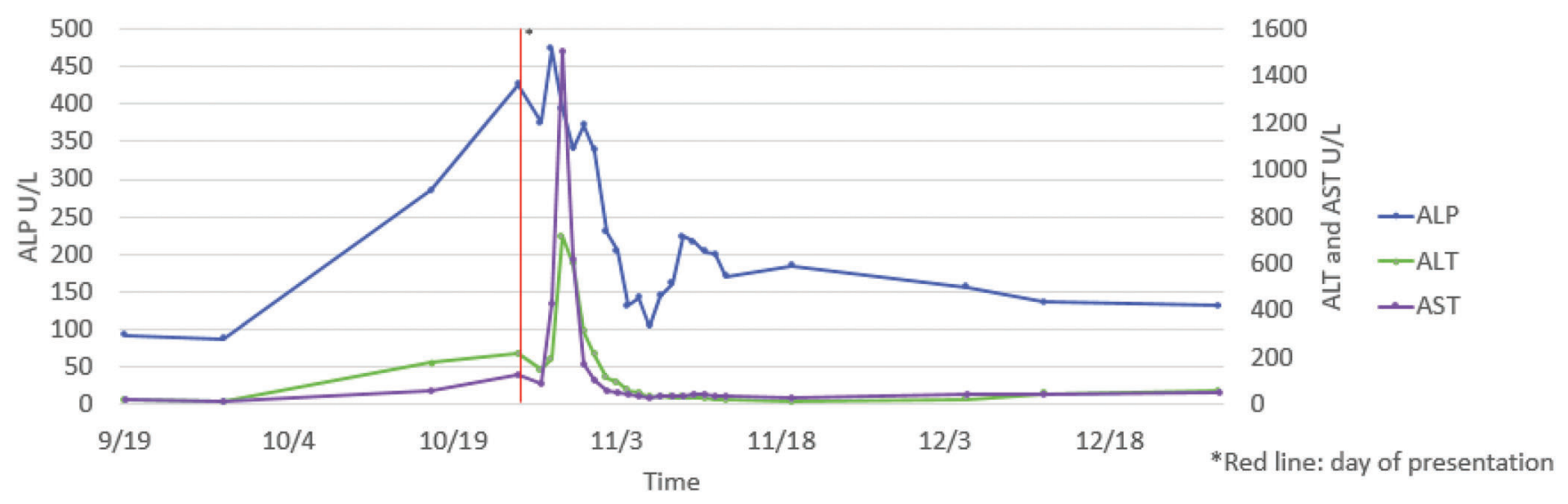

Fig. 1. Alkaline phosphatase (ALP), alanine aminotransferase (ALT), and aspartate aminotransfarse (AST) trends of the patient. Initial data points from office visits beginning September $19^{\text {th }}(9 / 19)$. Last data point from post-discharge follow-up data at the end of December (12/28).

areas of ulceration, nodularity, and pseudopolyps. Both the ileum and appendix were without significant abnormalities. There was no evidence of ischemia, dysplasia or granulomas in any section, and overall the findings were compatible with UC.

\section{Discussion}

Hepatic abscesses are an uncommon complication of IBD. To our knowledge, this is the youngest reported case of a hepatic abscess related to UC, with the next youngest in 18 year-old patients. A number of factors may have contributed to this early development. IBD patients with more frequent flares and higher IBD severity, as in our case, are at increased risk of hepatic abscesses. Higher inflammatory activity disrupts the barrier between the gut and the portal drainage, which may result in a portal bacteremia. Indeed, patients with UC have up to a $25 \%$ risk of portal bacteremia. ${ }^{1,2}$

Our patient's abscess cultures showed multiple gut bacteria, supporting that his bacteremia originated from the gut. As in our case, this bacteremia can develop into pylephlebitis or cause bacterial seeding into the liver. A suppurative

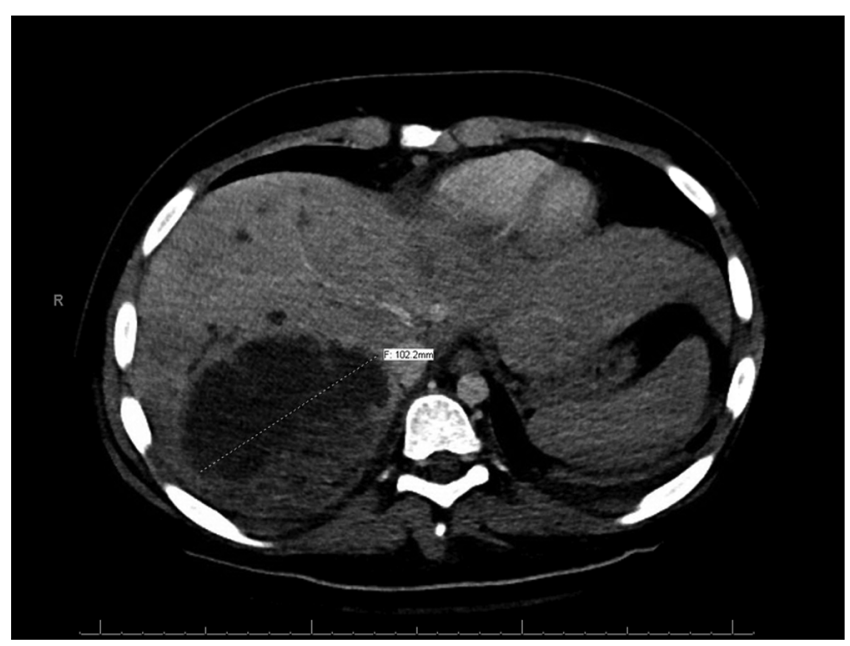

Fig. 2. Computed tomography (CT) of the abdomen with contrast done on admission. This axial view demonstrates the largest loculation the patient had, located in the right hepatic lobe. pylephlebitis can lead to a secondary portal vein thrombosis, ${ }^{3}$ which is likely the cause of thrombosis in our patient. While our patient was at increased risk of mycobacterial superinfections from the immunosuppression, which may also present as abscesses and hepatic complications, the acid-fast bacilli stain was negative. The patient's blood culture was also positive for S. viridans, which was not specified on any wound culture. This may have been due to streptococcal species being identified as skin flora on the aspirate culture, as there were Gram-positive cocci in pairs noted. It is also possible that the $S$. viridans was a contaminant, but given that this is a common species in the gastrointestinal tract (especially the oral cavity), it may again be evidence of significant bacterial gut translocation.

The patient's immunocompromised state likely contributed to the development of liver abscesses. He was not only on daily corticosteroids and intermittently on azathioprine but also had recently completed an induction course of infliximab. Additionally, infliximab may have potentiated a liver injury that promoted bacterial growth, as it can cause hepatotoxicity, autoimmune hepatocellular damage, and transaminitis in patients with UC. ${ }^{4,5}$ Azathioprine can also cause hepatotoxicity in up to $10 \%$ of IBD patients, and may promote venoocclusive disease, ${ }^{6}$ which our patient developed.

Our case highlights the importance of prompt recognition of a liver abscess, as it resulted in septic shock and a prolonged intensive care stay. Liver abscesses are associated with a high mortality rate, with one study showing a rate of $21 \%$, which worsens with delayed diagnosis and immunosuppressive treatment. ${ }^{7}$ Our patient also developed a portal vein thrombosis as a likely complication from his bacteremia, which can also carry a high mortality rate of up to $50 \%{ }^{6}$

Part of the diagnostic quandary is that many of the signs related to the presence of abscesses such as fevers and abdominal pain are similar to manifestations of IBD flares, potentially causing delays in diagnosis. A localized abdominal pain is not a reliable indicator of a liver abscess, as only five out of eight reported cases in UC patients with this complication had associated right upper quadrant abdominal pain. ${ }^{3}$ Our patient also presented with right shoulder pain, likely indicating diaphragmatic irritation; however, this has also not been consistently documented on prior reports. As these symptoms are either nonspecific or not reliably sensitive, lab work is the next reasonable evaluation. 
Our patient presented with significantly elevated ALP, transaminitis, and hyperbilirubinemia, all which support liver pathology. However, an elevated ALP is the single most reliable laboratory abnormality for the diagnosis of a liver abscess. ${ }^{8}$ Indeed, our patient's ALP was within normal limits at only 3 weeks before presentation. Accordingly, amongst relevant UC case reports, ALP was elevated in five out of six cases. Previous studies on patients with IBD propose an evaluation scheme to help guide in diagnosis of liver abscesses. ${ }^{9}$ If an IBD patient has a fever of unknown source, regardless of abdominal pain/tenderness, they should at least have their ALP checked, if not also their transaminases, bilirubin level, and CRP. Even if only the ALP is elevated, this is indicative of a liver pathology, and a CT of the abdomen can be used as a next step.

The treatment course of the abscess in our patient was not atypical when compared to other case reports, with both drainage and antibiotics resulting in improvement starting within weeks of therapy. Complete resolution is often achieved by 6 months, as in our own patient. Our patient went almost a year from admission being asymptomatic without any immunosuppressive therapy for his UC. This is particularly surprising as he was previously corticosteroiddependent, with IV corticosteroids providing only brief remission. There are a number of possible contributing factors. He did have approximately 1 month of parenteral nutrition and bowel rest due to his critical condition. Theoretically, total parenteral nutrition and bowel rest can induce remission in IBD by decreasing antigenic stimulation from food in the gut. However, there is only limited evidence of the therapeutic effect of total parenteral nutrition on IBD, and what is present supports that it can improve Crohn's disease but not UC. ${ }^{10-12}$ Total parenteral nutrition is therefore unlikely to be the primary therapeutic factor. Our patient did, however, continue exclusive enteral nutrition therapy that is recommended as a first-line treatment for remission in children with Crohn's disease. ${ }^{13}$ Exclusive enteral nutrition though has not been shown to be particularly effective in UC or colon-only Crohn's disease, ${ }^{14-17}$ so this is again unlikely to have been the primary cause of his prolonged remission.

Instead, we speculate that an immune recalibration away from autoimmunity due to the patient's septicemia may have played a part. Specific autoantibodies in UC indicate that the autoimmunity is at least partially mediated by colon-specific self-reactive $B$ cells. ${ }^{18,19}$ De novo self-reactive $B$ cells do not survive in germinal centers when their target self-antigen is expressed ubiquitously at or proximal to the germinal centers. ${ }^{20}$ Our patient had a significant bacteremia, as evidenced by the sepsis, pylephlebitis, and polymicrobial abscesses, indicating high gut permeability and capillary leakage even without evidence of a perforation. This could result in a significant number of colonic and bacterial proteins being released into the bloodstream, which in turn may trigger this ubiquitous exposure of these self-antigens that could induce antigenic tolerance. This transient recalibration of tolerance could have been potentiated by the patient's prior immunosuppression.

Azathioprine can trigger apoptosis of $\mathrm{T}$ cells, including potentially self-reactive memory-T cells. ${ }^{21}$ Furthermore, infliximab, as a TNF- $\alpha$ blocker, normalizes/increases the relative amount of pre-switched IgM B cells, leading to decreased antigen-specific antibodies. Indeed, this decrease in IgG antibodies before therapy is associated with improved rates of remission. ${ }^{18,22}$ Both the infliximab and azathioprine then may have helped to induce tolerance and remission by removing enough self-reactive immune cells, thus allowing the balance to shift towards new self-tolerant cells.

\section{Conclusions}

In summary, this is a rare, if not first, report of hepatic abscesses in a pediatric patient with UC. It is important to recognize signs of a hepatic abscess and risk factors for such, including immunosuppression and use of hepatotoxic agents, so as not to delay diagnosis. As hepatic abscesses can have severe consequences including sepsis, rapid diagnosis is optimal, and use of liver and inflammatory markers, particularly ALP, can guide the decision to image the patient with CT. This report also demonstrates a prolonged remission of steroid-dependent UC off any immunosuppressive therapies, the cause of which is unknown. We propose that our patient's immune system may have been re-calibrated away from autoimmunity through a series of events, including severe initial immunosuppression followed by significant release of self-antigen into the bloodstream from mucosal damage and permeability worsened by septic shock. These self-antigens may have become ubiquitous to de novo $B$ cells, causing the production of antigen-tolerant B cells. This case then raises questions about possible future modalities of UC treatment in the modulation of autoimmunity.

\section{Conflict of interest}

The authors have no conflict of interests related to this publication.

\section{Author contributions}

Contributed to study design, analysis and interpretation of data, manuscript writing, critical revision (DFC, RC, VG).

\section{References}

[1] Brooke BN, Slaney G. Portal bacteraemia in ulcerative colitis. Lancet 1958;1: 1206-1207. doi: 10.1016/s0140-6736(58)91910-x.

[2] Manousos ON, Doxiades T. Colitis and liver disease. Am J Proctol 1966;17: 48-51.

[3] Margalit M, Elinav $H$, Ilan $Y$, Shalit M. Liver abscess in inflammatory bowel disease: report of two cases and review of the literature. J Gastroenterol Hepatol 2004;19:1338-1342. doi: 10.1111/j.1440-1746.2004.03368.x.

[4] REMICADE (infliximab). Available from: https://www.accessdata.fda. gov/drugsatfda_docs/label/2013/103772s5359lbl.pdf.

[5] Ghabril M, Bonkovsky HL, Kum C, Davern T, Hayashi PH, Kleiner DE, et al. Liver injury from tumor necrosis factor- $\alpha$ antagonists: analysis of thirty-four cases. Clin Gastroenterol Hepatol 2013;11:558-564.e3. doi: 10.1016/j.cgh. 2012.12.025.

[6] Rojas-Feria M, Castro M, Suárez E, Ampuero J, Romero-Gómez M. Hepatobiliary manifestations in inflammatory bowel disease: the gut, the drugs and the liver. World J Gastroenterol 2013;19:7327-7340. doi: 10.3748/wjg.v19. i42.7327.

[7] Song J, Swekla M, Colorado P, Reddy R, Hoffmann S, Fine S. Liver abscess and diarrhea as initial manifestations of ulcerative colitis: case report and review of the literature. Dig Dis Sci 2003;48:417-421. doi: 10.1023/a: 1021964502199

[8] Rubin RH, Swartz MN, Malt R. Hepatic abscess: changes in clinical, bacteriologic and therapeutic aspects. Am J Med 1974;57:601-610. doi: 10. 1016/0002-9343(74)90012-6.

[9] Mir-Madjlessi SH, McHenry MC, Farmer RG. Liver abscess in Crohn's disease. Report of four cases and review of the literature. Gastroenterology 1986;91: 987-993. doi: 10.1016/0016-5085(86)90704-3.

[10] Hartman C, Eliakim R, Shamir R. Nutritional status and nutritional therapy in inflammatory bowel diseases. World J Gastroenterol 2009;15:2570-2578. doi: 10.3748 /wjg. 15.2570 . 
[11] Seo M, Okada M, Yao T, Furukawa $\mathrm{H}$, Matake $\mathrm{H}$. The role of total parenteral nutrition in the management of patients with acute attacks of inflammatory bowel disease. J Clin Gastroenterol 1999;29:270-275. doi: 10. 1097/00004836-199910000-00009.

[12] Wejdrychowicz A, Zajac A, Tomasik P. Advances in nutritional therapy in inflammatory bowel diseases: Review. World J Gastroenterol 2016;22: 1045-1066. doi: 10.3748/wjg.v22.i3.1045.

[13] Forbes A, Escher J, Hébuterne X, Kłek S, Krznaric Z, Schneider S, et al. ESPEN guideline: Clinical nutrition in inflammatory bowel disease. Clin Nutr 2017;36:321-347. doi: 10.1016/j.clnu.2016.12.027.

[14] Afzal NA, Davies S, Paintin M, Arnaud-Battandier F, Walker-Smith JA, Murch $\mathrm{S}$, et al. Colonic Crohn's disease in children does not respond well to treatment with enteral nutrition if the ileum is not involved. Dig Dis Sci 2005;50: 1471-1475. doi: 10.1007/s10620-005-2864-6.

[15] Alastair F, Emma G, Emma P. Nutrition in inflammatory bowel disease. JPEN ] Parenter Enteral Nutr 2011:35:571-580 doi: $10.1177 / 0148607111413599$.

[16] Lee D, Albenberg L, Compher C, Baldassano R, Piccoli D, Lewis JD, et al. Diet in the pathogenesis and treatment of inflammatory bowel diseases. Gastroenterology 2015;148:1087-1106. doi: 10.1053/j.gastro.2015.01. 007.
[17] Seidman EG. Nutritional management of inflammatory bowel disease. Gastroenterol Clin North Am 1989;18:129-155.

[18] Caneparo V, Pastorelli L, Pisani LF, Bruni B, Prodam F, Boldorini R, et al. Distinct anti-IFI16 and anti-GP2 antibodies in inflammatory bowel disease and their variation with infliximab therapy. Inflamm Bowel Dis 2016;22: 2977-2987. doi: 10.1097/MIB.0000000000000926.

[19] Takahashi F, Das KM. Isolation and characterization of a colonic autoantigen specifically recognized by colon tissue-bound immunoglobulin $\mathrm{G}$ from idiopathic ulcerative colitis. J Clin Invest 1985;76:311-318. doi: 10. 1172/JCI111963.

[20] Chan TD, Wood K, Hermes JR, Butt D, Jolly CJ, Basten A, et al. Elimination of germinal-center-derived self-reactive $B$ cells is governed by the location and concentration of self-antigen. Immunity $2012 ; 37: 893-904$. doi: $10.1016 / \mathrm{j}$. immuni.2012.07.017.

[21] Maltzman JS, Koretzky GA. Azathioprine: old drug, new actions. J Clin Invest 2003;111:1122-1124. doi: 10.1172/JCI18384.

[22] Timmermans WM, van Laar JA, van der Houwen TB, Kamphuis LS, Bartol SJ, Lam $\mathrm{KH}$, et al. B-cell dysregulation in crohn's disease is partially restored with infliximab therapy. PLoS One 2016;11:e0160103. doi: 10.1371/journal.pone. 0160103. 